\title{
Liderazgo y cultura organizacional como factores de influencia en la calidad universitaria: un análisis conceptual
}

\author{
Liliana M. Pedraja-Rejas*, Ítalo A. Marchioni-Choque, Constanza J. Espinoza-Marchant y Camila P. Muñoz-Fritis \\ Departamento de Ingeniería Industrial y de Sistemas, Facultad de Ingeniería Universidad de Tarapacá, 18 de \\ septiembre 2222, Arica- Chile. (correo-e: Ipedraja@uta.cl; imarchio@uta.cl; conniee.marchant@gmail.com; \\ camila.munoz.fritis@gmail.com) \\ * Autor a quien debe ser dirigida la correspondencia.
}

Recibido Oct. 19, 2019; Aceptado Dic. 11, 2019; Versión final Feb. 19, 2020, Publicado Oct. 2020

\begin{abstract}
Resumen
Este trabajo se ha planteado como objetivo realizar una revisión de la literatura sobre el liderazgo, la cultura organizacional y la calidad en las instituciones de educación superior con el fin de contribuir a la búsqueda, desde una perspectiva conceptual, de relaciones entre estas dimensiones. Esto debido a que el liderazgo y la cultura se consideran factores importantes para el desarrollo de habilidades y competencias en los graduados. Se recurre a artículos, preferentemente, de revisión conceptual y a estudios empíricos de revistas de corriente principal de los últimos años, cuyo análisis y su posterior integración, permitieron establecer un modelo explicativo a través de la interrelación de estas variables. Se concluye que es necesario el ejercicio de líderes activos y la promoción de culturas eficaces, ya que tienen el potencial de influir en el cumplimiento de los objetivos organizacionales y en el logro de la calidad institucional, afectando así la formación de profesionales competentes.
\end{abstract}

Palabras clave: liderazgo; cultura organizacional; calidad; educación superior; modelos de calidad

\section{Leadership and organizational culture as influencing factors in the quality of higher education: conceptual analysis}

\begin{abstract}
This article aimed to perform a literature review on leadership, organizational culture, and quality in higher education institutions to assess links between these dimensions and to contribute to their research from a conceptual perspective. Leadership and culture are considered important factors to develop skills and competences developments in graduates. Conceptual review articles and empirical studies from mainstream journals published in recent years were used to establish an explanatory model for the interrelationships between leadership, organizational culture, and quality. It was concluded that the employment of active for leaders and the promotion of effective cultures was needed since they had the potential to influence the fulfillment of organizational objectives and the achievement of institutional quality, which affected the formation of competent professionals.
\end{abstract}

Keywords: leadership; organizational culture; quality; higher education; quality models 


\section{INTRODUCCIÓN}

Actualmente, la educación superior se encuentra en un proceso turbulento y ad portas de una innovación disruptiva, tanto en el ámbito de la producción de conocimiento como en la docencia, es por esto que surge la importancia de que las universidades se preocupen de su capacidad reflexiva, del conocimiento que generan y transmiten y de su contribución social (Brunner et al., 2019). En este contexto, se reconoce que para responder a los desafíos actuales y futuros es cada vez más necesario un conjunto diferente de competencias, con mayor énfasis en el liderazgo colaborativo, la competencia intercultural global y la capacidad de responder ágilmente a las circunstancias cambiantes (Rubaii, 2016). Conscientes de esto, muchos países han invertido en la educación superior con el fin de garantizar diversidad, equidad y calidad para maximizar el potencial de los estudiantes, y conseguir así la capacidad de generar conocimiento e innovación, lo que a su vez impulse la productividad y el crecimiento económico de los países (Ferreyra et al., 2017).

Según Harvey y Green (1993) calidad posee diferentes significados para distintas personas, en este sentido, un mismo individuo puede adoptar distintas conceptualizaciones sobre la calidad en distintos contextos. En educación, calidad se puede referir a cualquiera de las diversas funciones de la universidad, incluidas sus actividades de investigación y participación comunitaria (McCowan, 2018). En efecto, la calidad en las instituciones de educación superior debe hacer referencia a la excelencia, desde la mirada en que la calidad se asocia al logro de los más altos estándares de exigencia, dificultad y complejidad, siendo fundamental considerar la consistencia entre los propósitos y el proyecto institucional (Pedraja et al., 2012).

Por otro lado, se reconoce que la calidad puede ser explicada, entre otros factores, por los procesos de gestión del conocimiento realizado en las distintas unidades académicas (Rodríguez-Ponce, 2016), los niveles de estrés de los docentes (Jawabri, 2017), el clima laboral (Rodríguez-Ponce y Pedraja-Rejas, 2017), la cultura organizacional (Trivellas y Dargenidou, 2009) y el liderazgo (Araneda-Guirriman et al., 2016). En estas últimas dos variables se centra el estudio, y es en este contexto, donde se reconoce que las instituciones de educación superior de clase mundial emergen en culturas académicas orientadas a la excelencia, es decir, culturas competitivas en las que los académicos tratan de alcanzar los máximos niveles en su trabajo (Pedraja-Rejas et al., 2018a). De igual manera, se ha concluido a partir de diversos estudios que la cultura organizacional en estas instituciones permite el tipo de reflexividad necesaria para que los administradores clarifiquen la identidad de esta, mientras que destacan sus cualidades únicas (Tierney y Lanford, 2018). Por otro lado, se ha descubierto que los estilos de liderazgo influyen en los niveles de satisfacción laboral (Torlak y Kuzey, 2019), además de tener el potencial de fomentar relaciones constructivas de los empleados, que junto a promover culturas de excelencia, permitiría el desarrollo de confianza entre estudiantes y la institución (Coman y Bonciu, 2016), resultando beneficioso para aumentar los niveles de productividad, eficiencia y calidad en el trabajo (Motilewa et al., 2015).

\section{METODOLOGÍA}

Considerando lo anterior, se opta por llevar a cabo una revisión documental sobre los estilos de liderazgo, los tipos de cultura organizacional y la calidad académica, con el fin de contribuir a la búsqueda, desde una perspectiva conceptual, de relaciones entre estas dimensiones en pos de avanzar en el reconocimiento de factores que ayuden a mejorar los niveles de calidad en las instituciones de educación superior. Para lograr esto, se recurre a fuentes secundarias, preferentemente a artículos de revisión conceptual y a estudios empíricos de revistas de corriente principal de los últimos cinco años, cuyo análisis profundo y minucioso, y su posterior integración, permitieron establecer un modelo explicativo a través de la interrelación de estas variables.

\section{REVISION DE CONCEPTOS}

Para poner el tema en el contexto del estudio, se presenta una breve revisión de los conceptos de liderazgo, cultura organizacional y calidad.

\section{Liderazgo}

A pesar de que el liderazgo ha sido un tópico de interés desde la antigüedad, este ha tomado fuerza desde el siglo XX, manteniéndose así hasta el día de hoy. En esta perspectiva y en el proceso de definición del concepto, el liderazgo ha sufrido cambios importantes, ya que en un principio las investigaciones de este eran realizadas desde una perspectiva individual, mientras que ahora es más bien considerada como una dinámica global, compleja, social y estratégica (Serrano y Portalanza, 2014). Es por esto que en la actualidad, el liderazgo es considerado como un proceso donde se ejerce una influencia en otras personas, a través de una 
comunicación efectiva, logrando así motivar a estos para contribuir en el logro de los objetivos del grupo o de una organización (Haslam y Reicher, 2016).

Entre los retos que tiene el efectivo ejercicio del liderazgo, es que el entorno de trabajo se ha vuelto mucho más complejo en términos de la naturaleza y la dinámica de los desafíos que enfrentan los líderes (Mohanty et al., 2016), por lo que estos deben ser flexibles para permitir así la adaptabilidad organizacional, como medida para sobrevivir y prosperar en el futuro. De igual manera, el proceso por el cual los seguidores ven el liderazgo no solo implica mecanismos organizativos formales (cargo u ocupación) sino también las redes informales que rodean a estos, ya que es necesario que el liderazgo sea construido socialmente, con el fin de que los líderes cuenten con la credibilidad y el reconocimiento de sus seguidores (Chiu, Balkundi y Weinberg, 2017).

Históricamente las empresas han buscado opciones para lograr mejores resultados, he ahí donde el manejo del liderazgo se ha alzado como una respuesta para alcanzar una ventaja competitiva, sin embargo, hoy en día se reconoce el papel de este como uno de los factores fundamentales para lograr el éxito organizacional sin restringirse solo al ámbito empresarial, ya que se incluyen otros sectores como los sociales, fuerzas armadas e instituciones educacionales. Bajo este escenario, diversos estudios indican que el liderazgo es considerado una herramienta que puede mejorar la eficacia y el desempeño en cualquier tipo de organización (Araneda-Guirriman et al., 2016), ya que este puede afectar los niveles de compromiso organizacional, satisfacción laboral y disposición a realizar tareas fuera de sus deberes correspondientes (Chiu, Balkundi y Weinberg, 2017).

Ahora bien, en el contexto de educación superior existen estudios empíricos que evidencian el hecho de que el liderazgo ejercido en esta área influye en los niveles de satisfacción laboral, en la flexibilidad organizacional (Rashid et al., 2016), en el manejo de conflictos, en el logro de la calidad (Araneda-Guirriman et al., 2016), y en el crecimiento, sustento y efectividad de la institución a largo plazo (Dey y Sood, 2018). Por lo tanto, desde el punto de vista conceptual, se entiende que el liderazgo va mucho más allá de la dirección hacia el cumplimiento de un objetivo, ya que un líder eficaz es quien influye en los trabajadores, interviniendo en el actuar de estos para así alcanzar el máximo desempeño en busca de la excelencia organizacional (Serrano y Portalanza, 2014).

\section{Los estilos de liderazgo}

Se han dedicado esfuerzos a distinguir las características y comportamientos de los líderes, estableciendo de esta forma distintos estilos de liderazgo. Es así como ha tomado mayor fuerza el modelo propuesto por Bass y Avolio, consolidándolos como unos de los mayores exponentes en cuanto a esta materia se refiere. Este modelo clasifica a los líderes en tres estilos: transformacional, transaccional y laissez-faire.

Los líderes transformacionales inspiran pasión y compromiso en sus seguidores y logran motivar a estos creando una visión atractiva, siendo un buen modelo a seguir y estableciendo metas desafiantes en un clima de respeto (Pedraja-Rejas et al., 2018b), con el fin de empoderar a sus subordinados para lograr alinear sus objetivos y los institucionales (Araneda-Guirriman et al., 2016). El liderazgo transformacional se compone de cinco factores, los cuales son: Influencia Idealizada (Atribuida y Conductual), aquí los subordinados convierten a sus líderes en modelos de referencia al considerar que las características y/o conductas exhibidas por estos son relevantes (Asrar-ul-Haq y Kuchinke, 2016); Motivación Inspiracional, referida a la capacidad del líder de inspirar y motivar a sus seguidores, promoviendo una visión común y creando un clima de confianza y mutuo entendimiento (Rodríguez-Ponce y Pedraja-Rejas, 2017); Estimulación Intelectual, basada en las características de los líderes para desafiar las ideas y valores de sus seguidores al resolver problemas (Jyoti y Dev, 2015), y por último, Consideración Individualizada, la cual ocurre cuando el líder se caracteriza por prestar atención a las necesidades individuales de sus subordinados, proporcionando empatía, un clima de apoyo y oportunidades de capacitación y aprendizaje (Niessen et al., 2017).

Por su parte, el liderazgo transaccional está más orientado a las tareas u objetivos que a las personas, y se basa principalmente en el intercambio equitativo entre líderes y seguidores centrándose en los intereses propios de ambos (Rosenbach et al., 2018). Asimismo, los líderes transaccionales clarifican las tareas, responsabilidades y roles de los seguidores, y los recompensan o castigan de acuerdo al desempeño que estos alcanzan (Yahaya y Ebrahim, 2016). Este estilo se compone de tres factores, los cuales son: Recompensa Contingente, donde los líderes se enfocan en definir claramente las tareas y se premia a los seguidores con recompensas materiales o inmateriales si cumplen con sus obligaciones (Jelača et al., 2016); Administración por Excepción Pasiva, en la cual el líder interviene únicamente ocurrido el error, y finalmente Administración por Excepción Activa, que ocurre cuando el líder monitorea activamente el progreso de los seguidores y aplica acciones correctivas cuando se detectan desviaciones para evitar que estas se vuelvan preocupaciones serias (Russell et al., 2018). 
Por último, el liderazgo laissez-faire se puede describir como un estilo no directivo y pasivo. Estos líderes son considerados indiferentes y, en consecuencia, puede ser difícil para los empleados establecer qué tan bien se desempeñan en sus tareas, o cuánto los respeta su líder (Van Prooijen y De Vries, 2015), creando así un ambiente de trabajo estresante para estos. Este estilo es la forma más inactiva e ineficaz de liderazgo, ya que el líder no se involucra en el desarrollo de sus subordinados, no otorga retroalimentación ni recompensas, no contribuye al crecimiento de los demás, no tiene iniciativa y no genera compromiso (Yahaya y Ebrahim, 2016). En base a lo antes expuesto y a modo comparativo, en la Tabla 1 se presenta un resumen de las principales características de los estilos de liderazgo transformacional, transaccional y laissez-faire planteados por Bass y Avolio.

Tabla 1: Resumen de los Estilos de Liderazgo

\begin{tabular}{|l|l|l|l|}
\hline Generalidades & Liderazgo Transformacional & Liderazgo Transaccional & Liderazgo Laissez-Faire \\
\hline $\begin{array}{l}\text { Características } \\
\text { principales de los } \\
\text { líderes }\end{array}$ & $\begin{array}{l}\text { Inspiradores, motivadores y } \\
\text { desafiantes. }\end{array}$ & $\begin{array}{l}\text { Prácticos, buscan } \\
\text { acuerdos entre todas las } \\
\text { partes. }\end{array}$ & $\begin{array}{l}\text { Evitan responsabilidades y la } \\
\text { toma de decisiones. }\end{array}$ \\
\hline $\begin{array}{l}\text { Contextos donde } \\
\text { estos líderes se } \\
\text { desempeñan mejor }\end{array}$ & $\begin{array}{l}\text { En situaciones estresantes y } \\
\text { de incertidumbre. }\end{array}$ & $\begin{array}{l}\text { En situaciones estables y y } \\
\text { rutinarias. }\end{array}$ & $\begin{array}{l}\text { Se suele asociar al estilo } \\
\text { más ineficaz, inactivo y que } \\
\text { causa mayor estrés en los } \\
\text { subordinados, por lo que no } \\
\text { se suele recomendar. }\end{array}$ \\
\hline Dimensiones & $\begin{array}{l}\text { Influencia Idealizada Atribuida, } \\
\text { Influencia Idealizada } \\
\text { Conductual, Motivación } \\
\text { Inspiracional, Estimulación } \\
\text { Intelectual y Consideración } \\
\text { Individualizada. }\end{array}$ & $\begin{array}{l}\text { Recompensa } \\
\text { Contingente, } \\
\text { Administración por } \\
\text { Excepción Pasiva y } \\
\text { Administración por } \\
\text { Excepción Activa. }\end{array}$ & - \\
\hline
\end{tabular}

Ahora bien, en relación a la educación superior se han hecho grandes descubrimientos en cuanto al efecto que tienen los estilos de liderazgo en este tipo de instituciones. Por ejemplo, Al-Mansoori y Koç (2019) al encuestar a docentes de dos escuelas de ingeniería de dos países distintos (Estados Unidos y Qatar), descubrieron que el estilo transformacional está altamente correlacionado con los niveles de satisfacción laboral. Asimismo, la investigación llevada a cabo por Akhtar et al. (2019) donde participaron 273 estudiantes de maestría de una universidad de China, arrojó que la percepción de este estilo tiene un efecto positivo en la comunicación y confianza entre los miembros del equipo de trabajo, lo que en consecuencia mejora la creatividad y el desempeño de estos. Por otra parte, Rodríguez-Ponce y Pedraja-Rejas (2017) realizaron un estudio en 38 unidades académicas de cuatro universidades chilenas, y descubrieron que dos dimensiones del estilo transformacional (influencia idealizada atribuida y estimulación intelectual) impactan de manera positiva sobre el clima orientado al servicio de los estudiantes, es decir, en el clima donde los docentes se preocupan por la preparación de clases, por los alumnos y por la calidad de los procesos educativos, lo que en consecuencia afecta de manera directa y significativa a la calidad de las carreras universitarias. Este último planteamiento se ve respaldo por el estudio llevado a cabo por Araneda-Guirriman et al. (2016), donde al analizar a 55 docentes de seis universidades del norte de Chile, descubrieron que el estilo transformacional es el que tiene mayor impacto favorable en la calidad de las carreras de pregrado.

En cuanto al liderazgo transaccional también existen estudios que sostienen el efecto positivo de este estilo en el contexto antes mencionado, por ejemplo, Torlak y Kuzey (2019) a través de una encuesta realizada a 189 trabajadores de instituciones de educación superior en Pakistán, descubrieron que la dimensión de administración por excepción perteneciente al estilo transaccional, tiene un efecto positivo y significativo con la satisfacción laboral y con el desempeño de los empleados. De igual manera la investigación de Dey y Sood (2018) donde participaron 215 docentes de diferentes instituciones de educación superior de India, arrojó que este estilo tiene un impacto significativo en la efectividad organizacional. Finalmente, para el caso del estilo laissez-faire los estudios no resultan muy auspiciosos en este contexto, ya que, por ejemplo, existen investigaciones como la de Bernasconi y Rodríguez-Ponce (2018), donde a partir de las opiniones de 56 líderes formales de cuatro instituciones chilenas de educación superior, se concluyó que este estilo es potencialmente negativo para el logro de la calidad en las titulaciones universitarias. De igual manera, Sadeghi y Pihie (2012) en su estudio donde encuestaron a 298 docentes de Universidades de Investigación de Malasia, descubrieron que el estilo laissez-faire se correlaciona negativamente con la efectividad del liderazgo, lo cual es considerado perjudicial ya que el éxito de los departamentos depende directamente de la efectividad de su líder, lo que a su vez cumple un papel importante en el éxito de la institución de educación superior. 


\section{Cultura organizacional}

El concepto de cultura organizacional ha sido ampliamente analizado en el ámbito de la antropología cultural y se ha vuelto popular tras descubrimientos que indican que esta tendría una incidencia directa sobre el desempeño organizacional, el comportamiento y la actitud de las personas dentro de una institución (Warrick, 2017). Numerosas conceptualizaciones han sido proporcionadas por los autores, sin embargo, se pueden encontrar varias similitudes entre estas. En efecto, la cultura de un grupo se puede definir como un patrón de suposiciones básicas compartidas y aprendidas al resolver sus problemas de adaptación externa e integración interna (Schein, 2004). En otras palabras, se puede decir que la cultura de una organización corresponde a los valores, filosofía, ideologías, creencias, expectativas, actitudes, normas y procesos compartidos dentro de cada una de ellas (Zhu, 2015). Dichos componentes sentarían la base de la comunicación y el entendimiento mutuo que determinaría el comportamiento de los empleados, así como sus procesos de integración y coordinación general (Naranjo-Valencia et al., 2016).

Se ha planteado que el poseer una cultura organizacional dominante puede conducir a una baja rotación de trabajadores, asimismo, esta posee un rol importante al influenciar y guiar el comportamiento de los empleados hacia el logro de los objetivos institucionales (Tang, 2017), logrando que estos sienten mayor confianza y compromiso hacia sus labores, lo que conlleva a su vez que mejore el desempeño de la organización como un todo. Por su parte, Ogbonna y Harris (2000) plantean que las organizaciones deberían orientar su cultura tanto en generar un enfoque externo como en crear cohesión interna, ya que centrarse solo en los valores compartidos dentro de estas podría reducir la rentabilidad a corto plazo, impedir el crecimiento e incluso afectar su supervivencia. Es por esto que, desde el punto de vista conceptual, se entiende que hacer de la capacitación cultural y la gestión efectiva de esta una prioridad puede traer una influencia significativa en el éxito y la ventaja competitiva de una organización (Warrick, 2017) sin importar el contexto al que pertenezca.

\section{Tipos de cultura organizacional}

Cameron y Quinn plantean el Modelo de Valores en Competencia, el cual ha sido uno de los más influyentes y utilizados en el área de investigación de la cultura organizacional (Yu y Wu, 2009). Este destaca dos dimensiones, una de estas contrapone la flexibilidad y la prudencia, frente a la estabilidad y el control, de esta manera algunas organizaciones son consideradas efectivas si tienden al cambio, es decir, aquellas que son adaptables y orgánicas, mientras que otras son vistas como efectivas mientras sean estables, predecibles y mecanicistas. Asimismo, la segunda dimensión contrapone un enfoque externo frente a uno interno, de tal forma que algunas organizaciones son vistas como efectivas si tienen características internas armoniosas, mientras que otras se consideran efectivas si estas se enfocan en interactuar o competir con otros fuera de sus límites (Cameron y Quinn, 2006; Naranjo-Valencia et al., 2016). Del modelo de Cameron y Quinn se desprenden cuatro tipos de cultura que se contraponen, la dimensión de clan se opone diagonalmente a mercado mientras que la dimensión adhocrática se opone diagonalmente a jerarquía (Cameron y Quinn, 2006). En la Tabla 2 se esquematizan los tipos de cultura planteados por Cameron y Quinn.

Tabla 2: Tipos de Cultura Organizacional

\begin{tabular}{|l|l|l|}
\hline Tipos de Cultura & Dimensiones & Características \\
\hline Cultura de Clan & $\begin{array}{l}\text { Flexibilidad y prudencia - enfoque } \\
\text { interno e integración. }\end{array}$ & $\begin{array}{l}\text { Flexibilidad, cooperación y trabajo en } \\
\text { equipo. }\end{array}$ \\
\hline Cultura Adhocrática & $\begin{array}{l}\text { Flexibilidad y prudencia - enfoque } \\
\text { externo y diferenciación. }\end{array}$ & Adaptabilidad e innovación. \\
\hline Cultura Jerárquica & $\begin{array}{l}\text { Estabilidad y control - enfoque interno } \\
\text { e integración. }\end{array}$ & $\begin{array}{l}\text { Responsabilidades, procesos y reglas } \\
\text { definidas. }\end{array}$ \\
\hline Cultura de Mercado & $\begin{array}{l}\text { Estabilidad y control - enfoque externo } \\
\text { y diferenciación. }\end{array}$ & $\begin{array}{l}\text { Competitivos y enfocados a los } \\
\text { resultados. }\end{array}$ \\
\hline
\end{tabular}

La cultura grupal o de clan obtiene este nombre debido a su semejanza a la estructura organizacional de una familia (Cameron y Quinn, 2006). En las organizaciones que poseen este tipo de cultura, las personas, propósitos y procesos se enfocan en la cooperación y la colaboración (Cameron y Quinn, 2011). Esta cultura enfatiza la flexibilidad, mientras que sus valores más importantes corresponden a la pertenencia, confianza y participación de sus miembros. Por otro lado, la cultura adhocrática o de desarrollo intenta fomentar la adaptabilidad, flexibilidad y la creatividad, asimismo la incertidumbre, ambigüedad y sobrecarga de información es típico en ellas (Cameron y Quinn, 2006). Las organizaciones que poseen cercanía a este tipo de cultura tienden a ser innovadoras, les agrada aceptar desafíos y responden rápidamente a los cambios del mercado. Asimismo, la cultura jerárquica o burocrática se caracteriza por poseer una estructura organizacional 
muy clara, reglas y procedimientos estandarizados, además de control estricto y responsabilidades bien definidas. Es por esto que las personas que pertenecen a estas organizaciones tienden a ser sistemáticas, cuidadosas y prácticas (Cameron y Quinn, 2011). Por último, la cultura racional u orientada al mercado se enfoca en las transacciones con entes externos en lugar de internos (Yu y Wu, 2009) y es debido a esta orientación de posicionamiento que estas organizaciones logran ser competitivas y productivas en el mercado (Cameron y Quinn, 2006). El lema de esta cultura es hacer las cosas rápidamente (Cameron y Quinn, 2011), por esta razón bajo esta tipificación, las organizaciones se caracterizan por ser competitivas, consistentes y tener respuestas rápidas (Naranjo-Valencia et al., 2016).

En el ámbito de la educación superior, existe evidencia que indica que la cultura organizacional tiene una incidencia en los niveles de calidad, como es el caso de Motilewa et al. (2015), donde a través de un estudio realizado en la Universidad de Covenant, se concluye que la cultura en estas instituciones también podría tener un efecto positivo en la motivación y la moral de los trabajadores, además de generar una buena voluntad que podría ser beneficioso para la productividad, eficiencia, calidad y actitud de estos en su lugar de trabajo, de la misma manera que permite fomentar una actitud creativa e innovadora en la institución, además de ayudar a que los estudiantes se desempeñen más eficientemente y con un mínimo de distracciones. Por otro lado, Daud et al. (2015) realizan un estudio en instituciones de alto y bajo desempeño en Malasia, descubriendo que para mejorar los niveles de desempeño es recomendable manifestar una cultura que comparta rasgos de los cuatro tipos (clan, adhocrática, mercado y jerárquica), sin embargo, Bendermacher et al. (2016) por su parte indican que a pesar de que los cuatro tipos de cultura forman una ventaja potencial en relación a criterios de efectividad, culturas como la jerárquica puede obstaculizar el desarrollo de la calidad. De igual manera, en un estudio realizado por Trivellas y Dargenidou (2009) en el Instituto Educacional Tecnológico de Larissa, se logró determinar que la cultura adhocrática mejora los niveles de calidad de servicio en la educación superior, ya que esta incluye el intraempren-dimiento, la experimentación, la creatividad, la proactividad, la adaptación y la innovación, los cuales corresponden a valores propicios para mejorar la calidad de la enseñanza y la administración.

\section{Calidad}

Calidad es un término bastante amplio, y cómo es posible aplicarla a diversas ramas de estudio, diferentes autores proponen interpretaciones distintas para su significado. Para Deming (1982), quien es considerado uno de los gurús de la calidad, esta representa un grado predecible de uniformidad, confiabilidad a bajo costo y adecuación al mercado, en otras palabras, calidad significa cumplir con las necesidades del consumidor. A pesar que el enfoque de la calidad estaba en sus orígenes principalmente orientado a la dirección de las organizaciones, en el último tiempo se ha consolidado la lógica de la calidad total en el ámbito de la educación superior, ya que en la actualidad se reconoce que una educación eficiente es un factor importante para el crecimiento económico de los países (Pedraja-Rejas et al., 2018a).

\section{Calidad en la educación superior}

Harvey (1995) asegura que calidad en la educación superior es un concepto relativo, el cual tiene significados diferentes según las personas y el contexto educacional, por esta razón las políticas de calidad deben adaptarse a las metas y objetivos de la institución, a la misión y a los stakeholders afectados (Varouchas et al., 2018). Asimismo, Harvey y Green (1993) en su artículo clásico destacan cinco dimensiones de calidad en la educación superior (González y Espinoza, 2008; Harvey, 1999; Rodríguez-Ponce y Pedraja-Rejas, 2017), las cuales corresponden a: 1) calidad como excepción: entiende la calidad como algo especial. Tradicionalmente la calidad se refiere a algo distintivo y elitista, mientras que en la educación se relaciona a la excelencia y al logro de metas muy altas, pero alcanzables. Implica altos estándares, excepcionalidad y mejora continua; 2) calidad como perfección o consistencia: considera la calidad como un resultado perfecto, basándose en las premisas de "cero defectos" y "hacer las cosas bien al primer intento". De alguna forma democratiza la calidad, por lo que piensa que esta puede ser alcanzada por todos; 3 ) calidad como aptitud para lograr un propósito: indica que se deben cumplir los requerimientos, necesidades o deseos del "cliente" de forma eficaz. En educación superior, una institución de calidad debe ser consistente con sus logros y su misión, visión y objetivos planteados; 4) calidad como valor agregado: entiende la calidad como el retorno a la inversión, es decir enfoca la calidad a la eficiencia económica. Si el producto puede ser alcanzado a un costo menor, o un producto mejor puede ser logrado por el mismo costo, entonces el servicio es de calidad; y 5) calidad como transformación: considera la calidad como un estado de cambio. Si se entiende desde el punto de vista educacional, se refiere a los logros y empoderamiento de los estudiantes, junto al desarrollo de conocimiento el cual enriquece a estos, existiendo una transformación de los mismos en profesionales y de la investigación en nuevo conocimiento.

Es importante medir, asegurar e incrementar la calidad en las instituciones, por esto el aseguramiento de la calidad es el proceso sistemático a través del cual se evalúan y verifican los insumos, productos y resultados 
con los estándares de calidad aceptados, con el fin de garantizar que las instituciones cuenten con las condiciones requeridas para realizar sus funciones académicas, manteniendo y mejorando la calidad en estas, además de asegurar la responsabilidad y facilitar la armonización de estándares en programas académicos, instituciones y sistemas (González y Espinoza, 2008). Asimismo, el proceso de acreditación de la calidad se desarrolla en torno a la gestión académica, la cual está asociada con la consecución y asignación de los recursos necesarios para llevar adelante el proyecto educativo y cumplir con las funciones universitarias contempladas en la misión de la institución. Esta además es obligatoria en el área de docencia de pregrado y electiva en las áreas de docencia de postgrado, investigación y extensión. De igual manera, esta no es permanente, debido a que se otorga por un periodo de tiempo definido, dependiendo de su viabilidad y consistencia con el proyecto institucional y docente (Pedraja-Rejas y Rodríguez-Ponce, 2015; Henríquez y Juri, 2018). Ahora bien, los procesos de evaluación de la calidad de las carreras universitarias en Chile han focalizado su medición en tres grandes criterios establecidos por la Comisión Nacional de Acreditación (CNA), a saber: los propósitos e institucionalidad, las condiciones de operación y los resultados junto a la capacidad de autorregulación. En cualquier caso, dichos criterios han significado en los últimos años un ordenamiento en el país (Pedraja-Rejas et al., 2018b). De igual manera, se plantea que el aseguramiento de la calidad se compone de tres fases: 1) la fase interna, la cual se refiere a un proceso de autoevaluación, ya sea a través de encuestas a los alumnos, revisión interna realizada por docentes pares, auditorías internas para evaluar los procedimientos de calidad, encuestas a recién graduados y a empleadores, etc.; 2) la fase externa, la cual incluye auditorías externas, revisión externa por pares evaluadores, entre otros (Harvey, 1999), y finalmente 3) el pronunciamiento de la comisión, el cual dictamina si se otorga o se niega la acreditación a la institución por un determinado periodo de años. Actualmente en Chile el proceso de acreditación es voluntario y desde su puesta en marcha se había constituido como requisito para las instituciones de educación superior que deseen acceder a financiamientos del estado. Asimismo, el proceso había involucrado la colaboración entre organismos del estado como la CNA, el propio establecimiento, docentes, estudiantes, agencias acreditadoras, etc. permitiendo asegurar el cumplimiento de un conjunto de exigencias mínimas que garantizan que las instituciones cuentan con las condiciones requeridas para realizar sus funciones académicas (González y Espinoza, 2008). No obstante, es importante mencionar que esta situación cambiará a partir del año 2020 debido a que la acreditación institucional será obligatoria para las instituciones de educación superior autónomas, así como también para la carrera de odontología.

A pesar de que se considera que los años de acreditación corresponden a la mejor "proxy" de calidad en la educación superior en Chile (Bernasconi y Rodríguez-Ponce, 2018), existen otros indicadores que de igual manera intentan cuantificarla a través de algún índice o medida, como es el caso de la tasa de titulación oportuna, la cual indica la proporción de estudiantes que logran terminar su formación profesional en el tiempo previsto por el plan de estudios más un margen de un año (Latorre et al., 2010). Considerando que uno de los atributos de calidad en la educación se relaciona con una eficiente gestión de recursos para el cumplimiento de metas, este indicador se alza como una buena medida para conocer los costos asociados a la formación profesional y la gestión de los activos de las instituciones de educación superior. Entendiendo que existen otros indicadores para cuantificar calidad en las instituciones, se presenta en la Tabla 3 un cuadro resumen con aquellos postulados en este trabajo.

Tabla 3: Resumen de los Indicadores de Calidad Educacional

\begin{tabular}{|l|l|l|l|}
\hline Indicador & $\begin{array}{l}\text { Dimensión que } \\
\text { cuantifica }\end{array}$ & Método de obtención & Escala \\
\hline $\begin{array}{l}\text { Años de } \\
\text { Acreditación }\end{array}$ & $\begin{array}{l}\text { Requisitos mínimos } \\
\text { exigidos. }\end{array}$ & $\begin{array}{l}\text { A través de la CNA, la cual considera } \\
\text { consistencia del proyecto educativo, } \\
\text { recursos, planes de acción y } \\
\text { capacidad para cumplir objetivos, } \\
\text { entre otros. }\end{array}$ & $\begin{array}{l}\text { Años, que varían entre 0 } \\
\text { (no acreditada) y 7 } \\
\text { (máximo de acreditación). }\end{array}$ \\
\hline $\begin{array}{l}\text { Tasa de Titulación } \\
\text { Oportuna }\end{array}$ & $\begin{array}{l}\text { Gestión de recursos } \\
\text { para el cumplimiento } \\
\text { de metas. }\end{array}$ & $\begin{array}{l}\text { Número de estudiantes titulados } \\
\text { dividido por el número de años que } \\
\text { dura la carrera de pregrado más 1. }\end{array}$ & $\begin{array}{l}\text { Porcentaje, donde el más } \\
\text { cercano a 100\% es mejor. }\end{array}$ \\
\hline
\end{tabular}

Por otro lado, la calidad de la educación se considera como un factor importante para obtener mejores resultados de aprendizaje, ya que un plan de estudio de buena calidad no solo prescribe lo que se debe enseñar y aprender en las diversas materias, sino que también incluye el esfuerzo de las instituciones para ayudar a los alumnos a desarrollar habilidades y competencias que se alineen con las demandas de los empleadores, del entorno y con su propia misión institucional (Woya, 2019). En este contexto, existen estudios que mencionan que la calidad del programa educativo si tiene una incidencia en la formación de profesionales competentes (Harry et al., 2018), es decir, profesionales que posean características y habilidades claves, tales como la capacidad de tomar la iniciativa, de solucionar problemas, de escuchar y de ser flexibles (Bhola y Dhanawade, 2013). Asimismo, Støren y Aamodt (2010) quienes recolectaron información de 36.000 
estudiantes graduados pertenecientes a 13 países en Europa, concluyeron que la calidad del programa educacional tiene un efecto significativo en el desempeño de estos, ya que los contenidos y comportamientos aprendidos durante su etapa de formación son los que se suelen utilizar en el lugar de trabajo. Es por lo anterior que se vuelve de suma importancia que las instituciones como parte de sus mecanismos para garantizar la calidad, realicen un monitoreo constante de sus planes de estudio (considerando las habilidades clave integradas) para así asegurar la mejora en la experiencia del aprendizaje del estudiante y futuramente la satisfacción del empleador (Brits, 2018).

\section{RESULTADOS}

A partir de la revisión documental llevada a cabo, en el Figura 1 se plantea un modelo en el que se establece que la calidad institucional se ve afectada, entre otras variables, por el liderazgo y la cultura organizacional presente en el lugar. En definitiva, el modelo propuesto plantea que el estilo de liderazgo ejercido, ya sea transformacional, transaccional o laissez-faire, como el tipo de cultura organizacional, ya sea de clan, adhocrática, de mercado o jerárquica, tienen el potencial de afectar la calidad de las instituciones de educación superior (la cual puede ser medida en años de acreditación, la tasa de titulación oportuna, entre otros índices). De igual manera se reconoce que la importancia de la calidad radica en su posible incidencia en la formación de profesionales competentes.

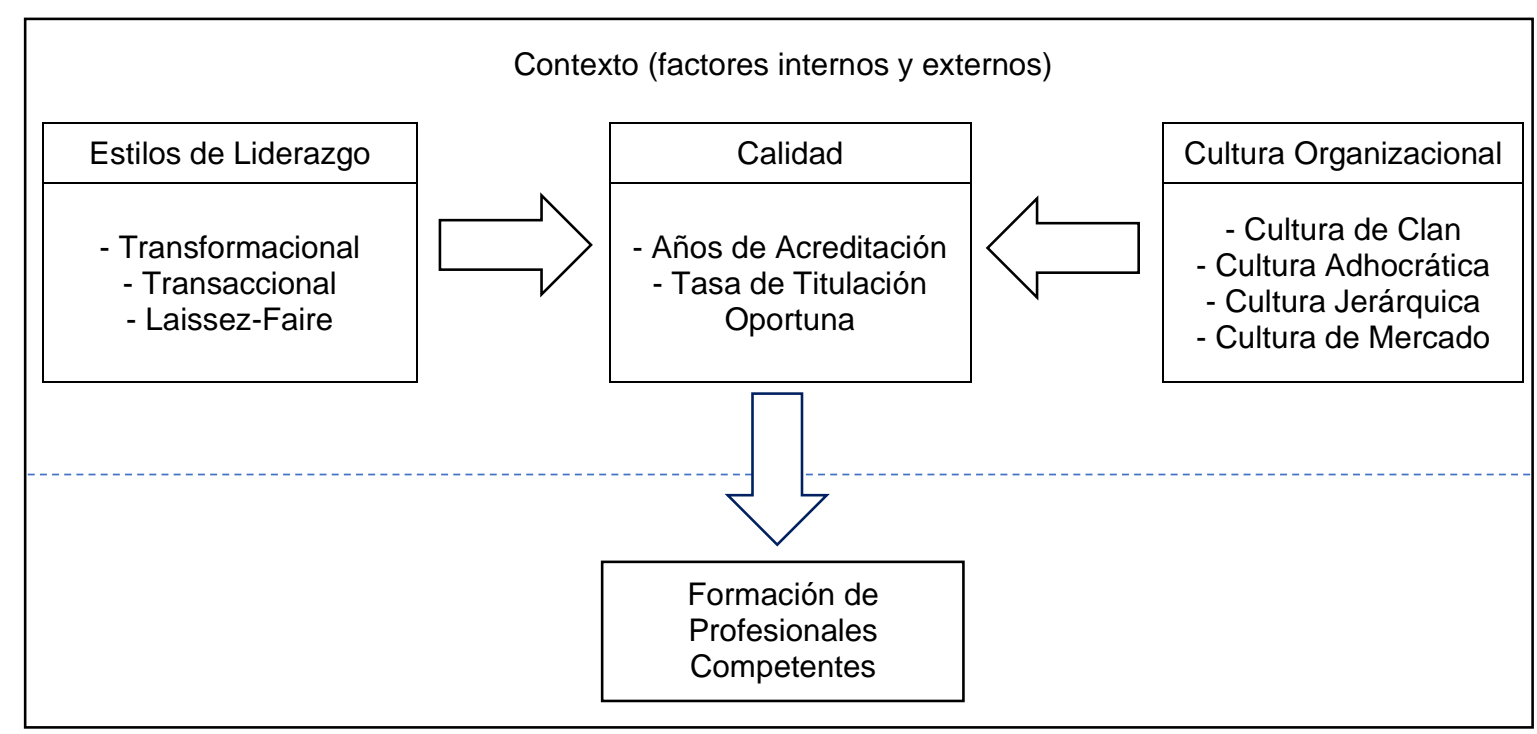

Fig. 1: Modelo Propuesto

El modelo propuesto puede ser explorado en estudios empíricos posteriores para determinar así el eventual efecto (positivo, negativo o indiferente) que tengan los estilos de liderazgo y el tipo de cultura en el logro de la calidad institucional, ya que a primera instancia uno podría creer, en base al análisis conceptual realizado, que el estilo transformacional sería el más óptimo para el logro del objetivo buscado, sin embargo, esto puede verse contradicho por el contexto en el que se encuentre. Lo mismo ocurre para el caso de la cultura organizacional, ya que se podría pensar que las culturas más flexibles y con rápida capacidad de adaptación a los cambios resultarían mejores para alcanzar los niveles de calidad deseados.

\section{DISCUSIÓN FINAL}

Cuando se habla de garantizar la calidad en una institución de educación superior, no solo se refiere a cumplir estándares mínimos de exigencias que diversos estados definen en la materia, sino más bien es un proceso que se construye a través del alineamiento de objetivos e intereses de diversos actores, como los institucionales, lo de los estudiantes y los de la sociedad en general, en donde para Chile, un país en vías de desarrollo, formar profesionales competentes podría ser la clave para mantenerse competitivos en un mundo cambiante y cada vez más orientado al conocimiento y la innovación. Es por esta razón que teniendo en cuenta que calidad en sí es un término complejo ya que depende de muchos factores, resulta interesante llevar a cabo un acercamiento teórico que permita entenderla para así avanzar en este tema.

Existen diversos autores que coinciden en que las variables de liderazgo y cultura organizacional tienen una relación con el logro de la calidad institucional, en el sentido de que afectan la eficacia, productividad y eficiencia de estas. Es por esto, que se reconoce que poner en el centro de la atención factores como la administración del liderazgo y el manejo de la cultura organizacional, podrían ayudar a mejorar la gestión de 
las universidades. Finalmente, el análisis conceptual realizado a partir de la revisión documental efectuada, permitió construir un modelo teórico de la calidad en las instituciones de educación superior, basada en los estilos de liderazgo (transformacional, transaccional y laissez-faire) y cultura organizacional (clan, adhocrática, de mercado y jerárquica) como factores de incidencia en el logro de esta. A pesar de que el modelo propuesto podría no capturar todos los factores que afectan la calidad institucional, ofrece la oportunidad de describir las relaciones entre variables, que, respaldadas por estudios empíricos previos, podrían afectar en mayor medida a la variable dependiente elegida.

\section{CONCLUSIONES}

De acuerdo al trabajo presentado y al análisis conceptual llevado a cabo, se pueden plantear las siguientes conclusiones principales: 1.- Se reconoce el potencial del liderazgo y de la cultura organizacional para impactar en los niveles de motivación, satisfacción y desempeño de los docentes. Por esto se hace necesario el ejercicio de líderes activos y la promoción de culturas eficaces que permitan el cumplimiento de los objetivos organizacionales. 2.- Se presentan estudios empíricos llevados a cabo en el campo de la educación superior que permiten evidenciar el efecto del liderazgo y la cultura organizacional en el logro de la calidad en este tipo de instituciones. 3.- La importancia de ofrecer una educación de calidad radica en el eventual impacto que tiene en la formación de profesionales competentes, lo que influiría a su vez, en la capacidad de los países para mantenerse competitivos en un mundo globalizado y de alta demanda de capital humano avanzado.

\section{AGRADECIMIENTOS}

Los autores agradecen el patrocinio recibido por parte de ANID a través del Proyecto Fondecyt 1170960 , titulado: La calidad en las instituciones de educación superior: la influencia de los estilos de liderazgo y la cultura académica. A su vez, se agradece el apoyo recibido del proyecto PIA CIE160007.

\section{REFERENCIAS}

Akhtar, S., Khan, K. U., y otros tres autores, Antecedents of Task Performance: An Examination of Transformation Leadership, Team Communication, Team Creativity, and Team Trust, https://doi.org/10.1002/pa.1927, Journal of Public Affairs, 19(2), 1-12 (2019).

Al-Mansoori, R. S., y Koç, M., Transformational Leadership, Systems, and Intrinsic Motivation Impacts on Innovation in Higher Education Institutes: Faculty Perspectives in Engineering Colleges, https://doi.org/10.3390/su11154072, Sustainability, 11(15), 1-26 (2019).

Araneda-Guirriman, C., Neumann-González, N., Pedraja-Rejas, L., y Rodríguez-Ponce, E., Análisis Exploratorio de las Percepciones sobre los Estilos de Liderazgo de los Directivos Universitarios en el Norte de Chile, http://dx.doi.org/10.4067/S0718-50062016000600013, Formación Universitaria, 9(6), 139-152 (2016).

Asrar-ul-Haq, M., y Kuchinke, K. P., Impact of Leadership Styles on Employees' Attitude Towards their Leader and Performance: Empirical Evidence from Pakistani Banks, https://doi.org/10.1016/j.fbj.2016.05.002, Future Business Journal, 2(1), 54-64 (2016).

Bendermacher, G., oude-Egbrink, M., Wolfhagen, I., y Dolmans, D., Unravelling Quality Culture in Higher Education: $A$ Realist Review, https://doi.org/10.1007/s10734-015-9979-2, Higher Education, 73(1), 39-60 (2016).

Bernasconi, A., y Rodríguez-Ponce, E., Análisis Exploratorio de las Percepciones sobre los Estilos de Liderazgo, el Clima Académico y la Calidad de la Formación de Pregrado, http://dx.doi.org/10.4067/S0718-50062018000300029, Formación Universitaria, 11(3), 29-40 (2018).

Bhola, S., y Dhanawade, S., Higher Education and Employability - A Review, http://dx.doi.org/10.2139/ssrn.2290103, SSRN Electronic Journal, 45-54 (2013).

Brits, H., Assessing Employer Satisfaction: An Attempt to Enhance Graduate Employability at an Institution of Higher Learning, https://doi.org/10.20853/32-5-2571, South African Journal of Higher Education, 32(5), 39-53 (2018).

Brunner, J. J., Labraña, J., Ganga, F., y Rodríguez-Ponce, E., Idea Moderna de Universidad: De la Torre de Marfil al Capitalismo Académico, https://doi.org/10.5944/educXX1.22480, Educación XX1, 22(2), 119-140 (2019).

Cameron, K. S., y Quinn, R. E., Diagnosing and Changing Organizational Culture: Based on the Competing Values Framework, Revised Edition, Jossey-Bass, San Francisco, USA (2006).

Cameron, K. S., y Quinn, R. E., The Competing Values Culture Assessment, A Tool from the Competing Values Product Line Diagnosing and Changing Organizational Culture: Based on the Competing Values Framework, $3^{\text {a }}$ Ed., Jossey-Bass, San Francisco, USA (2011).

Chiu, C.-Y., Balkundi, P., y Weinberg, F. J., When Managers become Leaders: The Role of Manager Network Centralities, Social Power, and Followers' Perception of Leadership, https://doi.org/10.1016/j.leaqua.2016.05.004, The Leadership Quarterly, 28(2), 334-348 (2017). 
Coman, A., y Bonciu, C., Organizational Culture in Higher Education: Learning from the Best, http://dx.doi.org/10.26417/ejser.v6i1.p135-145, European Journal of Social Sciences Education and Research, 6(1), 135145 (2016).

Daud, Y., Raman, A., y otros tres autores, The Type of Culture at a High Performance Schools and Low Performance School in the State of Kedah, http://dx.doi.org/10.5539/ies.v8n2p21, International Education Studies, 8(2), 21-31 (2015).

Deming, W., Quality, Productivity and Competitive Position, M. I. T Press, Cambridge, USA (1982).

Dey, P. P., y Sood, I., Impact of Leadership Styles on the Effectiveness of Higher Educational Institutions in Himachal Pradesh, OPUS: HR Journal, ISSN: 0973-9866, 9(1), 37-68 (2018).

Ferreyra, M., Avitabile, C., y otros tres autores, Momento Decisivo: La Educación Superior en América Latina y El Caribe. Resumen, Banco Mundial, Washington D. C., USA (2017).

González, L. E., y Espinoza, Ó., Calidad en la Educación Superior: Concepto y Modelos, https://doi.org/10.31619/caledu.n28.210, Calidad en la Educación, (28), 248-276 (2008).

Henríquez P., y Juri, H., Tendencias de la Educación Superior en América Latina y el Caribe 2018, Editorial UNC, Córdoba, Argentina (2018).

Harry, T., Chinyamurindi, W., y Mjoli, T., Perceptions of Factors that Affect Employability Amongst a Sample of Final-Year Students at a Rural South African University, https://doi.org/10.4102/sajip.v44i0.1510, SA Journal of Industrial Psychology, 44, 1-10 (2018).

Harvey, L., y Green, D., Defining Quality, https://doi.org/10.1080/0260293930180102, Assessment y Evaluation in Higher Education, 18(1), 9-34 (1993).

Harvey, L., Quality in Higher Education, Paper as the Swedish Quality Conference, 1-18, Göteborg, Suecia, Nov. (1999). Harvey, L., Beyond TQM, https://doi.org/10.1080/1353832950010204, Quality in Higher Education, 1(2), $123-146$ (1995). Haslam, S. A., y Reicher, S. D., Rethinking the Psychology of Leadership: From Personal Identity to Social Identity, https://doi.org/10.1162/DAED_a_00394, Daedalus, 145(3), 21-34 (2016).

Jawabri, A., Job Satisfaction of Academic Staff in the Higher Education: Evidence from Private Universities in UAE, International Journal of Human Resource Studies, ISSN: 2162-305, 7(4), 193-211 (2017).

Jelača, M., Bjekić, R., y Leković, B. A., Proposal for Research Framework Based on The Theoretical Analysis and Practical Application of MLQ Questionnaire, https://doi.org/10.1515/ethemes-2016-0028, Economic Themes, 54(4), 549-562 (2016).

Jyoti, J., y Dev, M., The Impact of Transformational Leadership on Employee Creativity: The Role of Learning Orientation, https://doi.org/10.1108/jabs-03-2014-0022, Journal of Asia Business Studies, 9(1), 78-98 (2015).

Latorre, M., Aravena, P., Milos, P., y García, M., Competencias Habilitantes: Un Aporte para el Reforzamiento de las Trayectorias Formativas Universitarias, https://doi.org/10.31619/caledu.n33.146, Calidad en la Educación, (33), 275-301 (2010).

McCowan, T., Quality of Higher Education in Kenya: Addressing the Conundrum, https://doi.org/10.1016/j.ijedudev.2017.11.002, International Journal of Educational Development, 60, 128-137 (2018).

Mohanty, J., Ara Begum, J., y Kar, B., Nuances of Leadership Effectiveness: Challenges of Context and Demographics, https://doi.org/10.21863/ij//2016.4.1.013, International Journal on Leadership, 4(1), 15-25 (2016).

Motilewa, D. B., Agboola, M. G., y Adeniji, C. G., Organizational Culture and Performance a case study of Covenant University, Nigeria, Covenant Journal of Business \& Social Sciences, ISSN: 2006-0300, 297-300 (2015).

Naranjo-Valencia, J. C., Jiménez-Jiménez, D., y Sanz-Valle, R., Studying the Links between Organizational Culture, Innovation, and Performance In Spanish Companies, https://doi.org/10.1016/j.rlp.2015.09.009, Revista Latinoamericana de Psicología, 48(1), 30-41 (2016).

Niessen, C., Mäder, I., Stride, C., y Jimmieson, N. L., Thriving When Exhausted: The Role of Perceived Transformational Leadership, https://doi.org/10.1016/j.jvb.2017.07.012, Journal of Vocational Behavior, 103, 41-51 (2017).

Ogbonna, E., y Harris, L. C., Leadership Style, Organizational Culture and Performance: Empirical Evidence from UK Companies, https://doi.org/10.1080/09585190050075114, International Journal of Human Resource Management, 11(4), 766-788 (2000).

Pedraja, L., Araneda, C., Rodríguez, E., y Rodríguez, J., Calidad en la Formación Inicial Docente: Evidencia Empírica en las Universidades Chilenas, http://dx.doi.org/10.4067/S0718-50062012000400003, Formación Universitaria, 5(4), 15-26 (2012).

Pedraja-Rejas, L., Araneda-Guirriman, C., Bernasconi, A., y Viancos, P., Liderazgo, Cultura Académica y Calidad de las Universidades: Aproximación Conceptual y Relaciones, Revista Venezolana de Gerencia, ISSN: 1315-9984, 23(1), 184199 (2018a).

Pedraja-Rejas, L., y Rodríguez-Ponce, E., Dirección Estratégica y Gestión en Instituciones de Educación Superior; in: A. Bernasconi (ed.), La Educación Superior en Chile: Transformación Desarrollo y Crisis, pp. 475-515, Ediciones UC, Santiago, Chile (2015). 
Pedraja-Rejas, L., Vega-Massó, R., y Riquelme-Castañeda, J., La Importancia de los Estilos de Liderazgo en la Calidad de las Unidades Académicas Universitarias, Revista Opción, ISSN: 1012-1587, 34(86), 130-151 (2018b).

Rashid, I. M. A., Samah, I. H. A., y otros siete autores, The Importance of Perceived Leadership Elements in Strategic Planning at Public University, https://doi.org/10.1016/s2212-5671(16)30343-4, Procedia Economics and Finance, 39, 422426 (2016).

Rodríguez-Ponce, E., y Pedraja-Rejas, L., Relación entre el Liderazgo Transformacional y el Clima Orientado al Servicio de los Estudiantes: Evidencia Exploratoria desde Chile, Interciencia, ISSN: 0378-1844, 42(10), 633-640 (2017).

Rodríguez-Ponce, E., Estudio Exploratorio del Impacto de la Gestión del Conocimiento en la Calidad de las Universidades, Interciencia, ISSN: 0378-1844, 41(4), 228-234 (2016).

Rosenbach, W. E., Taylor, R. L., y Youndt, M. A. (Eds), Contemporary Issues in Leadership, $7^{a}$ Ed., Routledge, New York, USA (2018).

Rubaii, N., Bringing the 21st-century Governance Paradigm to Public Affairs Education: Reimagining How We Teach What We Teach, https://doi.org//10.1080/15236803.2016.12002261, Journal of Public Affairs Education, 22(4), 467-482 (2016).

Russell, Z. A., Steffensen, D. S., y otros cuatro autores, High Performance Work Practice Implementation and Employee Impressions of Line Manager Leadership, https://doi.org/10.1016/j.hrmr.2018.02.003, Human Resource Management Review, 28(3), 258-270 (2018).

Sadeghi, A., y Pihie, Z. A. L., Transformational Leadership and Its Predictive Effects on Leadership Effectiveness, International Journal of Business and Social Science, e-ISSN: 2319-7064, 3(7), 186-197 (2012).

Schein, E. H., Organizational Culture and Leadership, 3ª Ed., John Wiley, Hoboken, USA (2004).

Serrano, B. J., y Portalanza, C. A., Influencia del Liderazgo sobre el Clima Organizacional, https://doi.org/10.1016/S2215910X(14)70026-6, Suma de negocios, 5(11), 117-125 (2014).

Støren, L., y Aamodt, P., The Quality of Higher Education and Employability of Graduates, https://doi.org/10.1080/13538322.2010.506726, Quality in Higher Education, 16(3), 297-313 (2010).

Tang, H., A Study of the Effect of Knowledge Management on Organizational Culture and Organizational Effectiveness in Medicine and Health Sciences, https://doi.org/10.12973/eurasia.2017.00700a, Eurasia Journal of Mathematics, Science and Technology Education, 13(6), 1831-1845 (2017).

Tierney, W., y Lanford, M., Institutional Culture in Higher Education, https://doi.org/10.1007/978-94-017-9553-1_544-1, Encyclopedia of International Higher Education Systems and Institutions, Dordrecht: Springer, 1-9 (2018).

Torlak, N. G., y Kuzey, C., Leadership, Job Satisfaction and Performance Links in Private Education Institutes of Pakistan, https://doi.org/10.1108/ijppm-05-2018-0182, International Journal of Productivity and Performance Management, 68(2), 276-295 (2019).

Trivellas, P., y Dargenidou, D., Organisational Culture, Job Satisfaction and Higher Education Service Quality, https://doi.org/10.1108/17542730910965083, The TQM Journal, 21(4), 382-399 (2009).

Van Prooijen, J. W., y De Vries, R., Organizational Conspiracy Beliefs: Implications for Leadership Styles and Employee Outcomes, https://doi.org/10.1007/s10869-015-9428-3, Journal of Business and Psychology, 31(4), 479-491 (2015).

Varouchas, E., Sicilia, M., y Sánchez-Alonso, S., Academics' Perceptions on Quality in Higher Education Shaping Key Performance Indicators, https://doi.org/10.3390/su10124752, Sustainability, 10(12), 1-16 (2018).

Warrick, D., What Leaders Need to Know about Organizational Culture, https://doi.org/10.1016/j.bushor.2017.01.011, Business Horizons, 60(3), 395-404 (2017).

Woya, A. A., Employability among Statistics Graduates: Graduates' Attributes, Competence, and Quality of Education, https://doi.org/10.1155/2019/7285491, Education Research International, 2019, 1-7 (2019).

Yahaya, R., y Ebrahim, F., Leadership Styles and Organizational Commitment: Literature Review, https://doi.org/10.1108/JMD-01-2015-0004, The Journal of Management Development, 35(2), 190-216 (2016).

Yu, T., y Wu, N., A Review of Study on the Competing Values Framework, https://doi.org/10.5539/ijbm.v4n7p37, International Journal of Business and Management, 4(7), 37-42 (2009).

Zhu, C., Organisational Culture and Technology-Enhanced Innovation in Higher Education, https://doi.org/10.1080/1475939X.2013.822414, Technology, Pedagogy and Education, 24(1), 65-79 (2015). 
